\title{
Matéria
}

ISSN 1517-7076

Revista Matéria, v. 11, n. 2, pp. 111 - 124, 2006

http://www.materia.coppe.ufrj.br/sarra/artigos/artigo10678

\section{Uma Metodologia para a Avaliação dos Gradientes de Tenacidade à Fratura ao Longo da Camada Cementada do Aço SAE 5115.}

\author{
${ }^{1}$ Leonardo Taborda Sandor, ${ }^{2}$ Itamar Ferreira \\ 1 Dpto. de Manufatura e Operações - Robert Bosch Ltda \\ CP: 1195. Robert Bosch Ltda, Campinas, SP. CEP 13012-970 \\ e-mail: leonardo.sandor@br.bosch.com \\ 2 Dpto. de Eng. de Materiais - Faculdade de Engenharia Mecânica - UNICAMP \\ CP: 6122. Cidade Universitária, Campinas, SP. CEP: 13083-970 \\ e-mail: itamar@fem.unicamp.br
}

\section{RESUMO}

Este trabalho propõe um modelo para avaliar pontualmente as variações de tenacidade à fratura ao longo da camada cementada de um aço SAE 5115. A pequena espessura dessas camadas impede a retirada de corpos de prova nas dimensões especificadas pelas normas de ensaios de tenacidade à fratura. Assim, para simular uma camada cementada retirou-se corpos-de-prova de tração e de tenacidade à fratura de amostras de aços SAE 5115, 5140, 5160 e 52100 assumindo a influência local apenas da variação do teor de carbono e considerando que os teores dos demais elementos de liga são essencialmente constantes. Os corpos-de-prova após eletrodeposição de cobre foram tratados termicamente em forno de cementação, têmpera e revenimento para serem submetidos aos efeitos térmicos sem absorção de carbono. Os resultados da análise microestrutural, dos ensaios de microdureza, de tração e de tenacidade à fratura foram agrupados em um único gráfico e comparados com o perfil de cementação de peças de aço SAE 5115 tratadas nas mesmas condições. Foi confirmado que a tenacidade à fratura varia inversamente proporcional à microdureza (HV1) e que a previsão do comportamento de uma trinca numa camada cementada pode ser feita por meio de equação ou diagrama que relacionam a microdureza HV1 com a tenacidade à fratura $\left(\mathrm{K}_{\mathrm{IC}}\right.$ ou CTOD $)$.

Palavras chaves: Tenacidade à Fratura, cementação, aço SAE 5115.

\section{A Model for Fracture Toughness Evaluation of the Carburized Layer for SAE 5115 Steel}

\section{ABSTRACT}

The purpose of this work is to propose a model for evaluating the fracture toughness along the SAE 5115 steel carburized layer. Due to the small thickness of those layers, it is impossible to machine specimens from those layer in accordance with standards. For simulating the microstructures of the carburized layer in order to get samples for tensile and the fracture toughness testing, specimens of SAE 5115, 5140, 5160, and 52100 steels have been machined, assuming the local influence just the variation of the content of carbon and considering that the contents of the other alloy elements are essentially constant. The specimens after electroplated copper layer were heat treated in an industrial load of carburizing, quenching, and tempering for they be submitted to the thermal effects without absorption of carbon. The results of the microstructure analysis and microhardness, tension, and fracture toughness tests were placed in a single graph and compared with the profile of the carburizing of the steel SAE 5115 heat treated in the same conditions. It was confirmed that the fracture toughness varies inversely proportional to the microhardness (HV1) and that the forecast of the behavior of a crack in a carburized layer can be made through equation or it designs that relate the microhardness (HV1) with the fracture toughness $\left(\mathrm{K}_{\mathrm{IC}}\right.$ or $\left.\mathrm{CTOD}_{\mathrm{C}}\right)$.

Keywords: Fracture toughness, carburizing, steel SAE 5115. 


\section{INTRODUÇÃO}

A indústria utiliza com freqüência peças de aços cementados, temperados e revenidos, visando obter alta resistência ao desgaste na superfície associada a um núcleo tenaz. Porém nos exames metalográficos para controle de qualidade do tratamento térmico, verifica-se esporadicamente a ocorrência de microtrincas (ou eventuais trincas) nas camadas cementadas dessas peças. Por desconhecer-se o comportamento ou estabilidade dessas trincas sob uma solicitação mecânica, muitas vezes, decide-se pelo sucateamento do lote inteiro de peças, com altos custos finais para os produtos fabricados. Em vista disso, o trabalho aqui desenvolvido propõe uma metodologia para avaliação dessas microtrincas e eventuais trincas, correlacionando microdureza HV1 com propriedades de tenacidade à fratura $\mathrm{K}_{\mathrm{IC}}$ e $\mathrm{CTOD}_{\mathrm{C}}$ visando prever a possibilidade de utilização dessas peças, com a segurança de qualidade necessária, evitando-se o sucateamento indiscriminado de peças.

Para a determinação da Tenacidade à Fratura de materiais metálicos, conforme os critérios estabelecidos nas normas ASTM E399-91(1997), E1290-02 e E1820-01 [1, 2, 3] ]. é necessário que nos corpos-de-prova a serem testados, sejam criadas trincas por fadiga, com comprimento mínimo de 2,5\% da largura do corpo de prova, e não inferior a 1,3 mm. Assim, essas normas não são aplicáveis na avaliação de corpos-de-prova que possuam camadas cementadas, pelo fato de existir um elevado gradiente de composição química na superfície do aço, nesses $1,3 \mathrm{~mm}$ mínimos especificados para a propagação de trinca por fadiga. Muitas vezes as espessuras das camadas cementadas são inferiores a este valor. Isto justifica a grande complexidade na análise da instabilidade de trincas superficiais existentes nas camadas cementadas de peças de aço.

A Figura 1 ilustra o perfil de carbono de uma camada cementada hipotética em aço SAE 5115 e compara a espessura dessa camada com o comprimento “a” da trinca por fadiga exigida para execução dos ensaios em conformidade com as normas acima. No caso ilustrado, a superfície tem teor de carbono de 1,0\%, e a região central não cementada tem $0,15 \%$. É possível visualizar que ao longo da camada cementada, pontualmente, as composições correspondem, a partir do núcleo, a aços SAE 5115, 5120, 5130,..., até atingir o SAE 51100 na superfície.



Figura 1: Variação do teor de carbono, comparação do comprimento “a” de uma trinca como função da profundidade da camada, e a hipótese de vários aços SAE 51xx da camada cementada.

Assim, a proposta para a avaliação da tenacidade à fratura ao longo da camada cementada, é caracterizar e analisar essa propriedade com composições químicas nominais correspondentes a vários aços SAE 51xx, ou seja, onde varie apenas o teor de carbono, e os demais elementos de liga possam ser considerados constantes. A seguir comparar a tendência dos valores obtidos nos ensaios, com a curva de cementação obtida para o SAE 5115, e tentar definir um comportamento para a tenacidade à fratura, em função da camada cementada. Neste trabalho foram utilizadas amostras de aços SAE 5115, 5140, 5160 e 51100, fabricados pela empresa Aços Villares SA, usina Anhangüera de Mogi das Cruzes - SP. Porém, como o aço SAE 51100 não é utilizado no Brasil, para esta pesquisa o mesmo foi substituído por SAE 52100. 
Assumiu-se que apenas o teor de carbono varia e que as variações dos teores dos demais elementos de liga possam ser desprezadas. Porém, esta simplificação não considera os efeitos das interações contínuas existentes numa camada cementada real, decorrentes do gradiente contínuo do teor de carbono e interfaces contínuas de transformação de fase e variações volumétricas. As peças cementadas são sujeitas ao fenômeno da fadiga, o que tem gerado de longa data diversos modelos matemáticos, onde alguns deles são comentados neste trabalho, e podem ser aplicados conjuntamente com a proposta aqui desenvolvida.

\section{MATERIAIS E MÉTODOS}

As amostras dos aços: SAE 5115, 5140, 5160 e 52100 foram retiradas a partir de barras laminadas pela usina siderúrgica Aços Villares - Mogi das Cruzes. A usinagem dos corpos-de-prova foi executada conforme especificado nas normas ASTM E8-01 e E1820-01 [3, 4]. Os corpos-de-prova foram revestidos com uma camada eletrodepositada de cobre de espessura $25 \mu \mathrm{m}$, para impedir a absorção e difusão de carbono da atmosfera cementante do forno de tratamento térmico. O tratamento térmico dos corpos-de-prova seguiu as diretrizes de UNTERWEISER [ㄷ] definidas para a série SAE 51XX e constou do seguinte:

- $\quad$ Cementação - $920^{\circ} \mathrm{C}$ por 180 minutos;

- $\quad$ Têmpera - $860^{\circ} \mathrm{C}$ por 60 minutos;

- $\quad$ Resfriamento em óleo - $60^{\circ} \mathrm{C}$ por 30 minutos;

- $\quad$ Revenimento $-160{ }^{\circ} \mathrm{C}$ por 90 minutos.

Os ensaios e análises desses corpos-de-prova foram feitos nas condições da tabela 1. O uso do aço SAE 52100 no lugar do SAE 51100 foi devido a este último não ser de uso corrente no Brasil, portanto não fabricado freqüentemente pela usina siderúrgica Aços Villares. A justificativa dessa substituição sem comprometimento do estudo é descrita no item 3.

Tabela 1: Condições dos ensaios e análises dos corpos de prova dureza, tração, tenacidade à fratura e metalográfica.

\begin{tabular}{|c|c|c|}
\hline Microdureza & ASTM E92-92 [ $\underline{6}]$ & Microdurômetro Shimadzu Type M carga de 9,81 N. \\
\hline Micrografia & ASTM E3-01 [7] & Analisador de imagens LEICA Quantimet $600 \mathrm{~S}$. \\
\hline Tração & ASTM E8-01 [4] & $\begin{array}{l}\text { Máquina Servo-Hidráulica MTS modelo } 810 \text { com } 100 \\
\text { kN e programa Teststar II da MTS. }\end{array}$ \\
\hline $\begin{array}{l}\text { Tenacidade à } \\
\text { Fratura }\end{array}$ & $\begin{array}{l}\text { ASTM E1820-01 } \\
{[\underline{3}]}\end{array}$ & $\begin{array}{l}\text { Máquina Servo-Hidráulica MTS modelo } 810 \text { com } 100 \\
\text { kN e programa Teststar II da MTS. Trinca de fadiga } \\
\text { obtida com razão de carga } \mathrm{R}=0,1 \text { aplicada a } 25 \mathrm{~Hz} \text {. }\end{array}$ \\
\hline Fractografia & & $\begin{array}{l}\text { Microscópio eletrônico de varredura JEOL modelo } \\
\text { JXA-840A. }\end{array}$ \\
\hline
\end{tabular}

\section{RESULTADOS E DISCUSSÃO}

As considerações sobre a metodologia aplicada, e os comportamentos observados são apresentadas separadamente sob a ótica das diferenças de composição química, respostas ao tratamento térmico (microestruturas) e propriedades mecânicas obtidas. Porém, como citado no item 1, esta simplificação não considera os efeitos das interações contínuas, existentes numa camada cementada real, decorrentes do gradiente contínuo do teor de carbono, interfaces contínuas de transformação de fase e variações volumétricas que resultam nas tensões residuais características das camadas cementadas. A simplificação fundamental deste estudo foi assumir que apenas o teor de carbono varia entre os diferentes tipos de aço, e que as variações dos teores de elementos de liga possam ser desprezadas. Foram utilizadas amostras de aços SAE 5115, 5140, 5160 e 52100, devido o 51100 não ser de uso corrente no Brasil. As conseqüências dessa substituição são analisadas a seguir e assumidas como não tendo influências significativas nos resultados.

Na revisão bibliográfica constatou-se que são poucos os trabalhos sobre tenacidade à fratura em camadas cementadas de aços, e a maioria estuda o comportamento em fadiga. Isto dificultou este estudo, devido a escassez de dados comparativos de tenacidade à fratura. Mas os autores aqui citados registram também grandes dificuldades em seus estudos de fadiga, devido a instabilidade do crescimento de trinca de fadiga na camada cementada. 


\subsection{Composição Química}

A Tabela 2 apresenta as composições químicas dos quatro aços estudados. As justificativas para o uso de aço SAE 52100 no lugar do SAE 51100, devido à indisponibilidade desse último, são discutidas a seguir analisando as influências das diferenças dos teores de elementos de liga (no caso manganês e cromo) na temperabilidade, na morfologia dos carbonetos de ferro e cromo, e no potencial de carbono da atmosfera cementante.

- A aplicação das equações para cálculo dos Fatores de Liga de temperabilidade definidas na norma SAE J406 [8], nas composições indicadas na tabela 2, apresentaram uma diferença de 13\%, o que se assumiu como não sendo significativo.

- A morfologia dos carbonetos de Cr e Fe na presença do Mn analisados segundo HUME-ROTHERY [9] permite assumir que como os aços SAE 51xx e 52xx têm cerca de $97 \%$ de ferro na sua composição e o restante constituído de carbono, cromo e manganês, as diferenças observadas na tabela 2 nos teores de cromo e manganês, não resultem em grandes diferenças de composição e morfologia dos carbonetos observados. O níquel não é influi na formação de carbonetos. E ainda, segundo REED-HILL [10] a dureza da martensita dos aços de baixa liga (menos de cerca de 5\% de elementos de liga), pode ser admitida como dependente apenas da concentração de carbono.

- A correlação entre os elementos de liga e o potencial de carbono da atmosfera cementante, definida pela equação (1) de LARSSON et al [11], resulta em $\mathrm{C}_{\mathrm{p}} / \mathrm{C}$ de 0,94 para o aço SAE 51XX, e 0,91 para o SAE 52XX, diferença esta assumida também como não significativa.

$\log \frac{C_{P}}{C}=0,055 .(\% S i)-0,013 .(\% M n)-0,040 .(\% C r)+0,014 .(N i)-0,013 .(\% M o)$

Tabela 2: Resultados das composições dos aços SAE 51XX e 52100 do estudo.

\begin{tabular}{|c|c|c|c|c|c|c|}
\hline & \multicolumn{2}{|c|}{ Especificação SAE J404-Jun/00 [12] } & \multicolumn{4}{|c|}{ Resultados das Análises } \\
\hline & SAE 51XX & SAE 52100 & SAE 5115 & SAE 5140 & SAE 5160 & SAE 52100 \\
\hline C & Obs. 1 & $0,98-1,10$ & 0,16 & 0,39 & 0,60 & 0,98 \\
$\underline{\text { Mn }}$ & $\underline{0,70-0,90}$ & $\underline{0,25-0,45}$ & $\underline{0,80}$ & $\underline{0,78}$ & $\underline{0,92}$ & $\underline{0,32}$ \\
P & máx. 0,030 & 0,025 & 0,020 & 0,016 & 0,013 & 0,010 \\
S & máx. 0,030 & 0,025 & 0,005 & 0,005 & 0,006 & 0,012 \\
Si & $0,15-0,35$ & $0,15-0,35$ & 0,27 & 0,23 & 0,22 & 0,23 \\
Cr & $\underline{0,70-0,90}$ & $\underline{1,30-1,60}$ & $\underline{0,81}$ & $\underline{0,80}$ & $\underline{0,82}$ & $\underline{1,46}$ \\
$\mathrm{Ni}$ & máx. 0,25 & max. 0,25 & 0,20 & 0,08 & 0,08 & 0,10 \\
$\mathrm{Mo}$ & máx. 0,06 & máx. 0,06 & 0,04 & 0,03 & 0,04 & 0,04 \\
$\mathrm{Al}$ & não especif. & não especif. & 0,018 & 0,019 & 0,018 & 0,031 \\
$\mathrm{Cu}$ & max. 0,35 & max. 0,35 & 0,16 & 0,16 & 0,13 & 0,20 \\
\hline
\end{tabular}

Obs. 1 - SAE 5115, C = 0,13 a 0,18; SAE 5140, C =0,38 a 0,43; SAE 5160, C = 0,56 a 0,64.

Obs. 2 - SAE 5160 especifica $\mathrm{Cr}=0,75$ a 1,00, mas aqui está na faixa de $0,70-0,90$.

\subsection{Microdureza HV1 e Microestrutura}

Os resultados de microdureza HV1 são mostrados na figura 2 sobrepostos ao perfil de dureza obtido em engrenagens de aço SAE5115 cementadas na mesma carga de forno industrial de tratamento térmico em que se trataram os corpos de prova. Verifica-se que há boa concordância entre os pontos, além dos resultados estarem de acordo com a publicação AMERICAN SOCIETY FOR METALS Handbook Volume 1 [13].

As microestruturas foram analisadas com base na observação feita em microscopia ótica de reflexão, correlação com diagramas de transformação sob resfriamento contínuo de ATKINS [14] e informações sobre tratamento térmico segundo UNTERWEISER [] d definidas para a série de aços SAE 51xx. Este critério foi assumido como sendo uma boa aproximação para interpretar o que ocorre na formação de camada cementada 
de um aço SAE 5115. Com relação às microestruturas, são válidas as seguintes considerações referentes às figuras 3 a 6 :

- $\quad$ SAE 5115 - predomina estrutura de bainita revenida. Na Figura 3 não são vistas as agulhas características da martensita revenida.

- $\quad$ SAE 5140 - predominância de estrutura de martensita revenida como revelam as agulhas na Figura 4, mesclada com alguma bainita.

- $\quad$ SAE 5160 - predominância de estrutura de martensita revenida como mostra a Figura 5 mesclada com alguma bainita similar ao aço SAE 5140.

- $\quad$ SAE 52100 - predominância de estrutura de martensita revenida mesclada com bainita, e também com carbonetos pró-eutetóides, devido ao teor de carbono ser superior a 0,77\% como mostra a Figura 6.

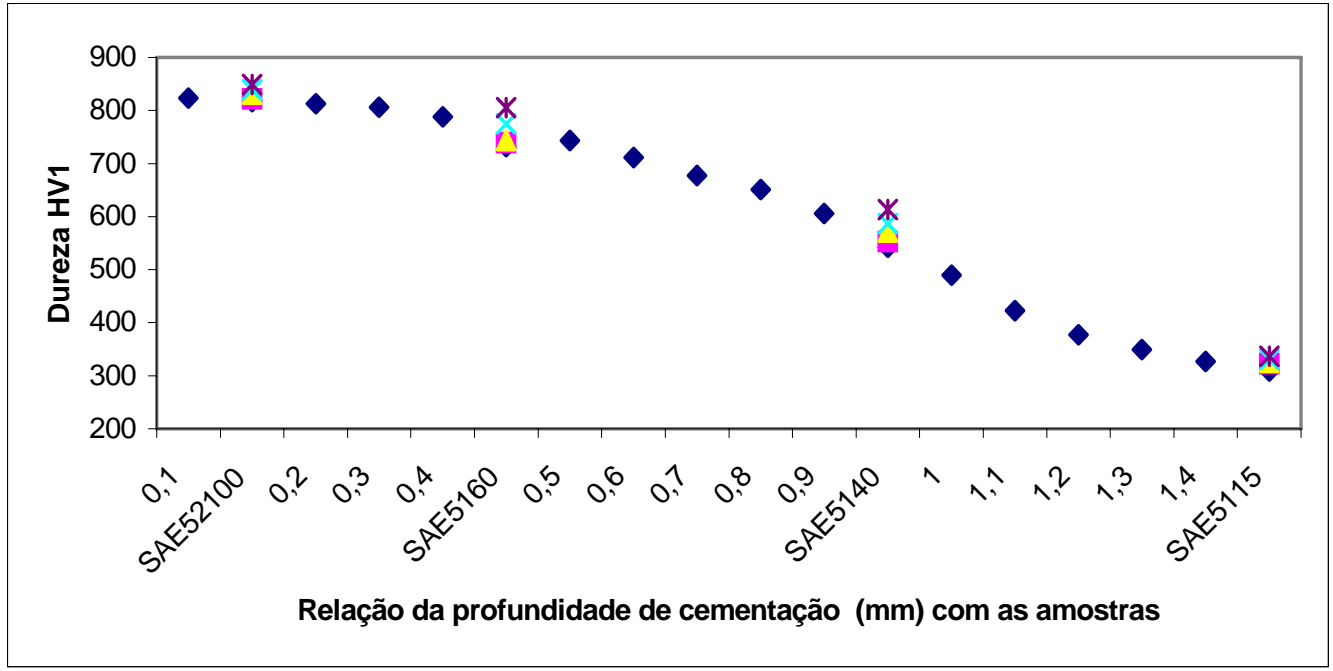

Figura 2: Sobreposição dos resultados de durezas das amostras dos aços, com o perfil de dureza de cementação obtido para engrenagens de aço SAE 5115, da mesma carga de tratamento térmico.

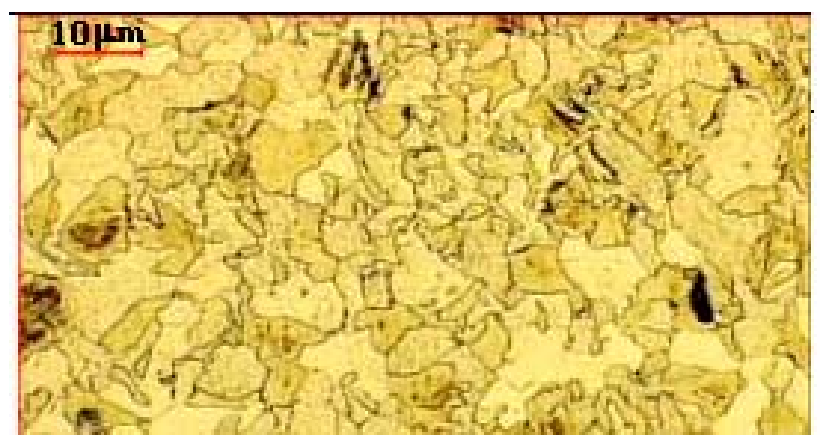

Figura 3: Micrografia do aço SAE 5115 após ataque com Nital 3\%, com aumento 1000 X. 


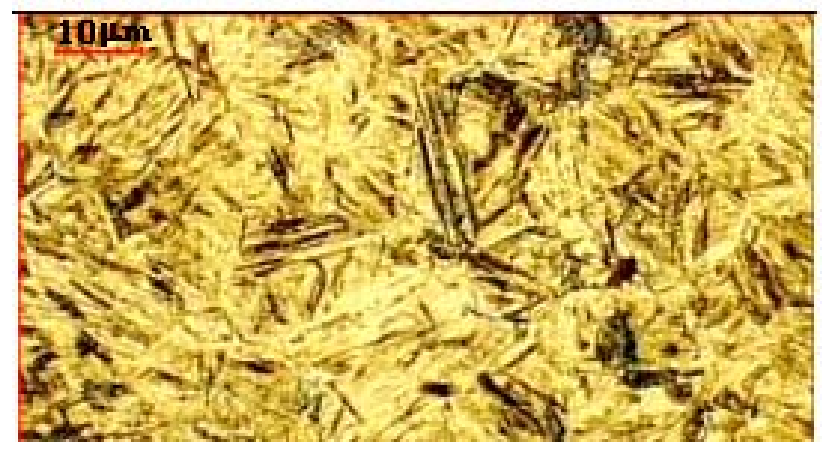

Figura 4: Micrografia do aço SAE 5140 após ataque com Nital 3\%, com aumento 1000 X.

NAGAMURA [15] propôs um método analítico para predição da vida em fadiga de engrenagens cementadas com intensa rede de carbonetos nas superfícies. Esse método considera que a trinca origina na superfície cementada composta de carbonetos + martensita na superfície, martensita abaixo da superfície e martensita de baixo carbono fora da camada cementada (no núcleo). $\mathrm{O}$ autor verificou que a trinca se propaga devido a fratura por clivagem dos carbonetos e fratura transgranular dos grãos de martensita. O comportamento em fadiga dos carbonetos mostrados na figura 6 pode ser entendido através do estudo de SHIN [16], que correlacionou estruturas de dois aços diferentes com as respectivas vidas em fadiga, para aplicação de molas tipo diafragma utilizado em embreagens de automóveis. Para tal foram comparados aços similares ao SAE 5150 e SAE 1085. No SAE 1085 é considerável a quantidade de carbonetos pró-eutetóides não dissolvidos, que atuam como descontinuidades para nucleação de trincas de fadiga. ERDOGAN [17] estudou o efeito do tamanho das partículas de martensita nas propriedades de tração de corpos de prova de aço SAE 8620 cementados com 0,80\% de carbono, com estrutura bifásica no núcleo não cementado. Em seu experimento, os corpos-de-prova de tração após cementação, foram temperados em temperaturas entre $\mathrm{Ac}_{3} \mathrm{e}$ $\mathrm{Ac}_{1}$ do material base, de modo que fosse obtida estrutura totalmente martensítica na superfície, e no núcleo uma mistura de cerca de $25 \%$ de martensita e $75 \%$ de ferrita. Concluiu que corpos-de-prova com microestrutura bifásica no núcleo exibiram resistência à tração e limite de escoamento levemente inferior e ductilidade superior, sem comprometer a dureza superficial, quando comparado com corpos-de-prova com núcleos praticamente temperados pelos tratamentos térmicos convencionais.

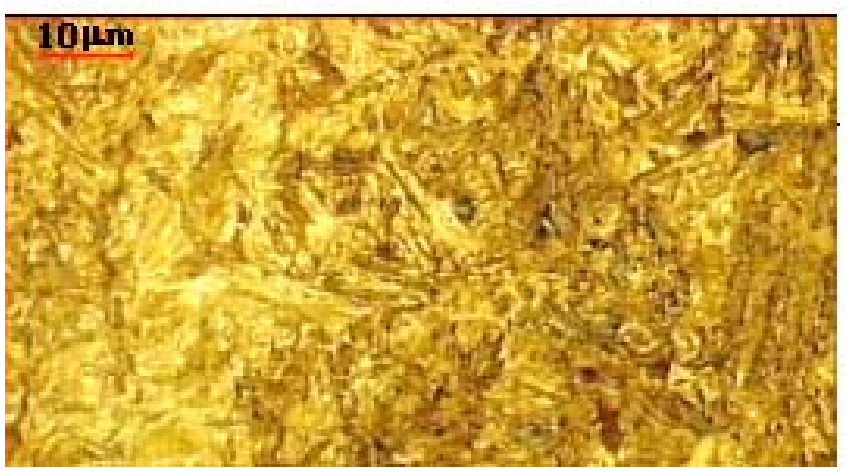

Figura 5: Micrografia do aço SAE 5160 após ataque com Nital 3\%, com aumento 1000 X. 


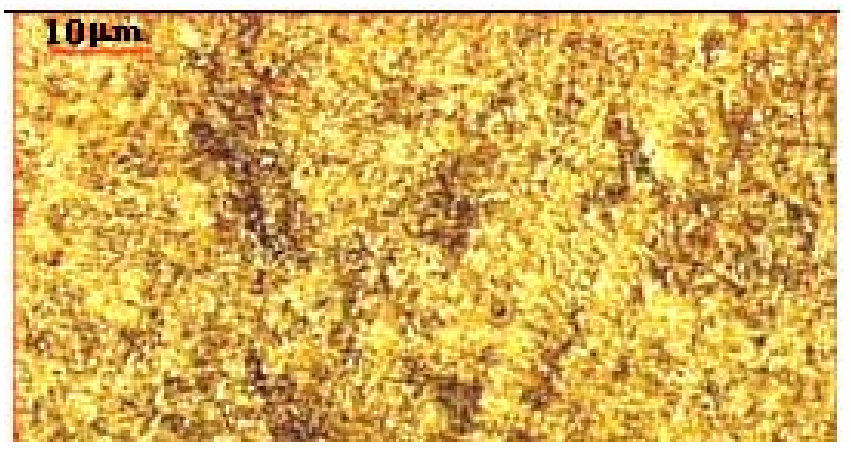

Figura 6: Micrografia do aço SAE 52100 após ataque com Nital 3\%, com aumento 1000 X.

\subsection{Tenacidade à Fratura $\left(\mathrm{K}_{\mathrm{IC}} \mathrm{e} \mathrm{CTOD}\right)$}

A Tabela 3 apresenta os resultados de tenacidade à fratura $\mathrm{K}_{\mathrm{IC}}$ e CTOD e microdureza HV1 obtidos para os aços SAE 5115, 5140, 5160, e 52100 e que neste estudo representam pontos discretos dentro de uma camada cementada. Verifica-se a diminuição dos valores de $\mathrm{K}_{\mathrm{IC}}$ e CTOD quando se aumenta a microdureza HV na posição em questão de uma suposta camada cementada. E através de regressão numérica, é possível obter dois polinômios que relacionam $\mathrm{HV}$ x $\mathrm{K}_{\mathrm{IC}}$ e $\mathrm{HV}$ x $\mathrm{CTOD}_{\mathrm{C}}$ que são representados pelas equações (2) e (3) e respectivos gráficos mostrados nas figuras 7 e 8 respectivamente. Isso significa que para o aço SAE 5115 cementado, temperado e revenido nas condições indicadas no item 2, é possível estimar o $\mathrm{K}_{\mathrm{IC}}$ e o $\mathrm{CTOD}_{\mathrm{C}}$ em função da microdureza HV1 obtida na camada cementada. A equação (2) é válida para microdureza superior a $550 \mathrm{HV} 1$, sendo que este valor define a profundidade efetiva da camada cementada, conforme norma DIN EN ISO 2639 [18]. E a equação (3) é válida para durezas a partir de 300 HV1 que é a dureza característica do núcleo.

- Microdureza HV1 com $\mathrm{K}_{\mathrm{IC}}$.

Tabela 3: Resultados de $\mathrm{K}_{\mathrm{IC}}$, CTOD, e HV1 para os aços SAE 5115, 5140, 5160 e 52100 determinados em conformidade com a norma ASTM E1820-01 [3] .

\begin{tabular}{|c|c|c|c|c|c|}
\hline & & SAE 5115 & SAE 5140 & SAE 5160 & SAE 52100 \\
\hline $\begin{array}{c}\mathrm{K}_{\mathrm{IC}} \\
\left(\mathrm{MPa}^{1 / 2}\right)\end{array}$ & $\bar{X}$ & 71 & 40 & 22 & 22 \\
\cline { 2 - 6 } & $\mathrm{S}$ & 1,6 & 1,5 & 1,9 & 2,8 \\
\hline $\begin{array}{c}\mathrm{CTOD}_{\mathrm{C}} \\
(\mathrm{mm})\end{array}$ & $\bar{X}$ & $1,17 \mathrm{E}-01$ & $2,12 \mathrm{E}-02$ & $4,29 \mathrm{E}-03$ & $1,94 \mathrm{E}-03$ \\
\cline { 2 - 6 } & $\mathrm{S}$ & $8,83 \mathrm{E}-02$ & $1,93 \mathrm{E}-02$ & $3,93 \mathrm{E}-04$ & $6,05 \mathrm{E}-04$ \\
\hline $\begin{array}{c}\text { Microdureza } \\
(\mathrm{HV} 1)\end{array}$ & $\bar{X}$ & 324 & 613 & 756 & 832 \\
\cline { 2 - 6 } & $\mathrm{S}$ & 11 & 29 & 31 & 13 \\
\hline
\end{tabular}

$$
K_{I C}=138,25 \cdot(H V 1)^{-3,41 \cdot 10^{-4} \cdot(H V 1)}
$$

Com coeficiente de correlação $\mathrm{R}=0,991$ e desvio padrão $\mathrm{S}=3,82 \mathrm{MPa} \cdot \mathrm{m}^{1 / 2}$.

- Microdureza HV1 com CTOD C.

$$
\mathrm{CTOD}_{C}=1,07 \cdot 10^{-3} \cdot(H V 1)^{-1,1 \cdot 10^{-3} \cdot(H V 1)}
$$

Com coeficiente de correlação $\mathrm{R}=0,968$ e desvio padrão $\mathrm{S}=1,64 \cdot 10^{-5} \mathrm{~m}$. 


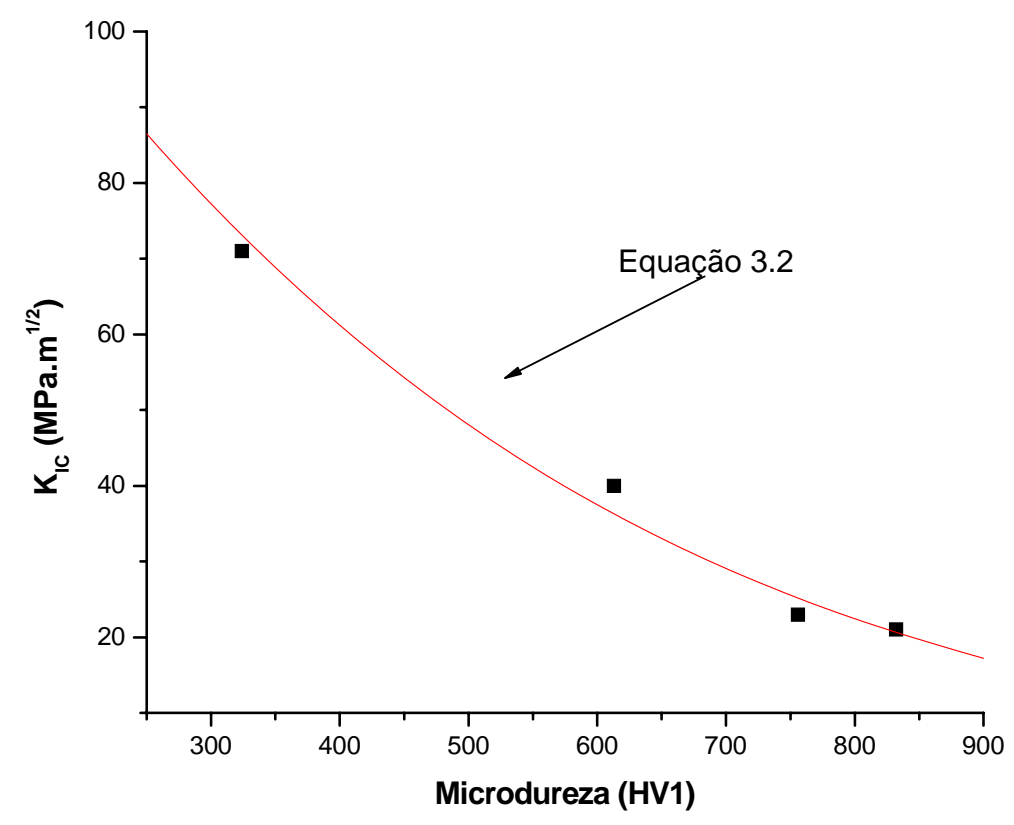

Figura 7: Variação de $\mathrm{K}_{\mathrm{IC}}$ em função da microdureza HV1 na camada cementada hipotética. O valor 350 HV1 seria o núcleo não cementado e 850 HV1 seria a superfície cementada.

O crescimento de trincas por fadiga em aços cementados, é de difícil análise devido às pequenas espessuras das camadas. As pesquisas de outros autores têm abordado vários aspectos no entendimento da nucleação e crescimento de trincas nas camadas cementadas no ponto de vista de fadiga, mas poucos pesquisam sobre o ponto de vista da mecânica da fratura. OBATA et al [19] avaliaram a taxa de crescimento de trinca por fadiga em aços cementados, através da técnica de emissão acústica, onde sensores acústicos são montados nas extremidades das amostras testadas. Com esse método é possível estimar a taxa de crescimento de trinca da/dN e ainda determinar as regiões de fratura intergranular da camada cementada e transgranular do material do núcleo. COHEN [20] estudou a resistência à fadiga comparando corpos-de-prova de aços SAE 4320 com cantos vivos e com cantos arredondados, que foram tratados termicamente simultaneamente, visando estimar o quanto que a geometria dos corpos de prova afeta a microestrutura e a resistência à fadiga de um aço cementado. Verificou que partículas de carbonetos grosseiros e austenita retida em excesso foram predominantes nos corpos-de-prova com cantos vivos, devido à não uniformidade da distribuição de carbono nesses cantos. KATO et al [21] propuseram um método de simulação de crescimento de trinca por fadiga num dente de engrenagem de aço cementado, baseado na mecânica de fratura. A vida em fadiga foi assumida como sendo log-normal, onde as resistências à fadiga foram estimadas pelo método escada, com a construção de curvas $\mathrm{S}-\mathrm{N}$ de probabilidades de falha 10,50 e $90 \%$. A média de resistência à fadiga para os grupos de engrenagens avaliados, variou de 875 a 1135 MPa, onde as engrenagens de menor módulo tiveram os maiores valores. 


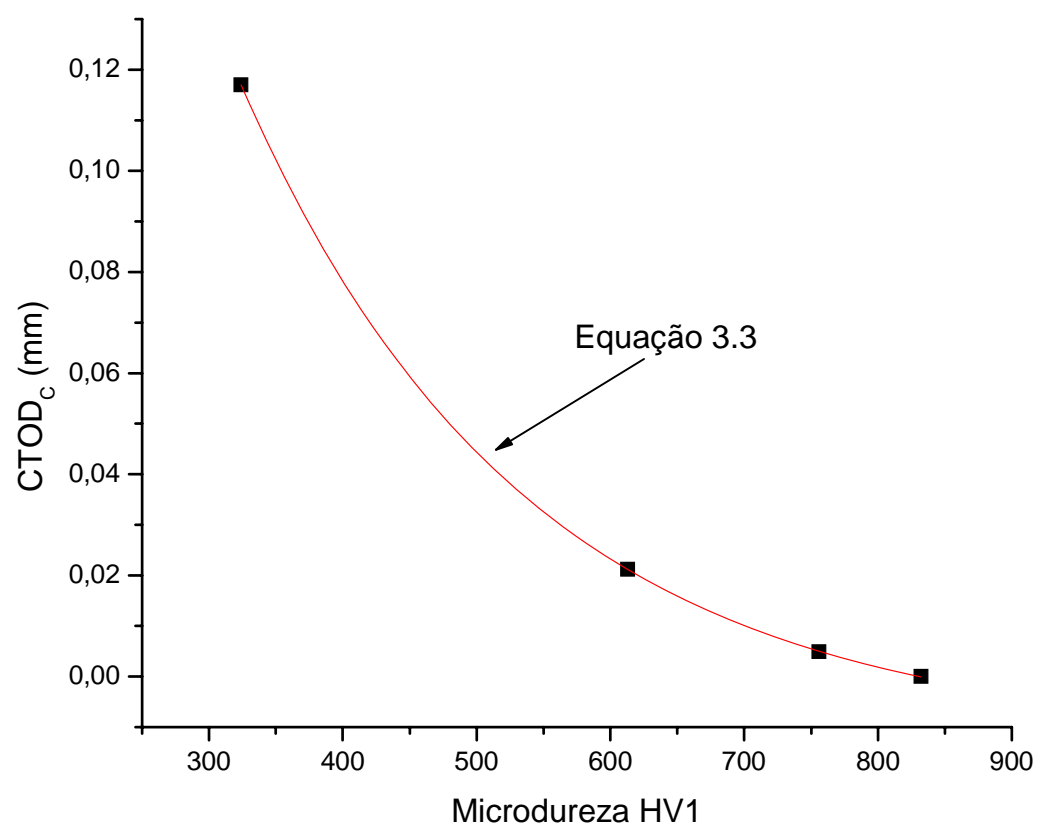

Figura 8: Variação de CTOD em função da microdureza HV1 na camada cementada hipotética. O valor 350 HV1 seria o núcleo não cementado e 850 HV1 seria a superfície cementada.

PRESTON [22] estudou a nucleação da trinca por fadiga e seu crescimento em corpos-de-prova de aço similar ao SAE 8615 com a superfície cementada, sob flexão rotativa. Verificou que acima de certo comprimento de trinca, o limite de fadiga é controlado por $\Delta \mathrm{K}_{\text {th }}$ e pela pré-existência de alguma trinca. Determinou experimentalmente o valor de $\Delta \mathrm{K}_{\mathrm{th}}=4 \mathrm{MPa} \sqrt{m}$ para estruturas martensíticas revenidas abaixo de $200{ }^{\circ} \mathrm{C}$, tornando possível estimar o limite de fadiga para diferentes comprimentos de trinca usando a equação experimental (4):

$$
K_{\text {máx }}=1,13 \cdot \sigma_{n} \cdot \sqrt{\pi \cdot a}
$$

onde:

a é o comprimento da trinca normal à superfície cementada;

$\sigma_{\mathrm{N}}$ é a tensão nominal (soma algébrica da tensão residual e a tensão de flexão externa aplicada);

$\mathrm{K}_{\text {máx }}$ (na flexão rotativa o $\mathrm{K}_{\text {mín }}<0$, então em substituição ao $\Delta \mathrm{K}$ utiliza-se o $\mathrm{K}_{\text {máx }}$ uma vez que o valor negativo não influencia na trinca).

E para a camada cementada de aço similar ao SAE 8615, determinou a equação (5) para controle de da/dN.

$$
\frac{d a}{d N}=9,93 \cdot 10^{-10} \cdot\left(0,4 \cdot\left(1,13 \cdot \sigma_{N} \cdot \sqrt{\pi \cdot a}\right)\right)^{2,02}
$$

LARSSON et al [111] baseados no trabalho de PRESTON [22], estudaram as propriedades de fadiga após cementação, verificaram que os resultados dos limites de fadiga variam com a dureza do material do núcleo não cementado atingindo um máximo de 920 MPa para 360 HV. No mesmo estudo, LARSSON et al [11] apresentam um modelo para predição do limite de fadiga, baseado na mecânica de fratura elástica linear, que permite interpretar as diferenças entre os limites de fadiga de diferentes aços através de diagramas. Estes diagramas correlacionam o limite de fadiga intrínseco como sendo aproximadamente 1,6 vezes a dureza Vickers do material, e a equação de Paris (6) do mesmo material. Um exemplo do diagrama é mostrado na figura 9. 


$$
\Delta K_{t h}=f \cdot \Delta \sigma \cdot \sqrt{\pi \cdot a}
$$

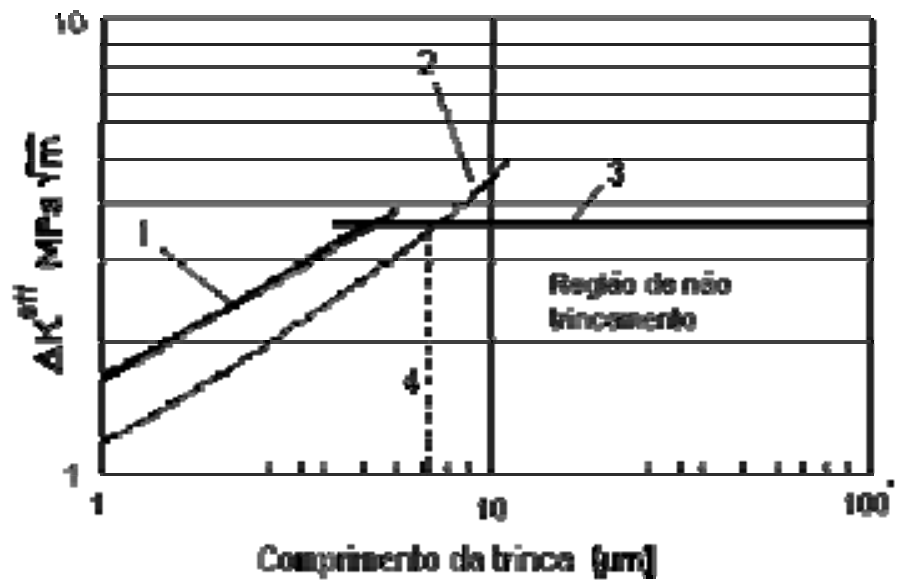

\section{I- Linite de finces perquess \\ 2- Fina da inbensidede de benotio ofitive 3- Linile de timesas bengest 4. Conpoinento inicial retiveo de trinea}

Figura 9: Relação entre comprimento da trinca e limite de fadiga de LARSSON et al [11]. Onde a reta 1 é definida por $\Delta K_{t h}=f \cdot 1,6 \cdot(H V) \cdot \Delta \sigma \cdot \sqrt{\pi \cdot a}$, e a reta $3 \Delta K_{t h}^{\text {eff }}=4 \mathrm{MP} \sqrt{\mathrm{m}}$.

Porém foi usado o $\Delta \sigma_{\text {eff }}$, que é a parte do $\Delta_{\sigma}$ que está abrindo a trinca, para calcular o $\Delta \mathrm{K}_{\text {eff q }}$ que por sua vez é a parte do $\Delta \mathrm{K}$ que abre a trinca com ajuda da equação (6). Fazendo-se isso, a avaliação não é influenciada pela razão de carga R, podendo-se utilizar para qualquer condição de razão de carga R. Como a trinca somente é aberta quando submetida a tensões de tração, o $\Delta \sigma_{\text {eff }}$ é definido pelas seguintes condições:

- $\Delta \sigma_{\text {eff }}=\sigma_{\max }-\sigma_{\min }$, quando $\sigma_{\min }>0$;

- $\Delta \sigma_{\text {eff }}=\sigma_{\max } \quad$ quando $\sigma_{\min }<0$;

- $\Delta \sigma_{\text {eff }}=0 \quad$ quando $\sigma_{\max }<0$.

Onde $\sigma_{\max } \mathrm{e} \sigma_{\min }$ correspondem às tensões aplicadas somadas algebricamente às possíveis tensões residuais que possam estar atuando.

GENEL [23] estudou os efeitos da profundidade da camada cementada sobre a performance em fadiga para o aço SAE 8620. Comparou corpos de prova temperados e revenidos, com outros cementados, temperados e revenidos, variando-se as espessuras das camadas cementadas. Verificou que quanto maior a relação t/D entre a espessura (t) da camada cementada e o diâmetro (D) da peça cementada e também quanto maior a relação "área da camada cementada dividida pela área do núcleo não cementado" $\left(A_{R}\right)$, maior será o limite de fadiga. A partir desses resultados, GENEL [23] desenvolveu algumas relações que permitem determinar o limite de fadiga do aço SAE 8620, como função dos valores t/D e $A_{R}$, de acordo com as equações (7) e (8).

$$
\begin{aligned}
& S_{f}=590+36400 \cdot(t / D)^{1,92} \quad, \text { em MPa } \\
& S_{f}=590+1040 \cdot\left(A_{R}\right)^{1,40}, \text { em MPa }
\end{aligned}
$$

MASUYAMA et al [24] desenvolveram uma equação que permite estimar o limite de fadiga por flexão nos dentes na camada cementada da engrenagem $\left(\mathrm{S}_{\mathrm{f}}\right)$, com relação à razão de carga $(\mathrm{R})$, a dureza $(\mathrm{HV})$ da superfície da camada cementada e a área projetada $\mathrm{S}$ do defeito na direção normal à aplicação da carga, de acordo com a equação (9). 
$S_{f}=\beta \cdot \frac{(H V+120)}{(\sqrt{S})^{1 / 6}} \cdot\left[\frac{(1-R)}{2}\right]^{\alpha}$

$\alpha=0,226+H V \times 10^{-4}$

$\beta=0,98$, sem descarbonetação e com trinca criada por fadiga;

$\beta=1,17$, com descarbonetação e sem trinca criada por fadiga;

$S=\sqrt{10} \times \delta$, onde $\delta$ é espessura da descarbonetação e sem trinca criada por fadiga;

$S=\sqrt{10} \times a$, sem descarbonetação e com trinca criada por fadiga ;

$a$ é o comprimento da trinca;

$\mathrm{S}_{\mathrm{f}}$ é o limite de fadiga por flexão (nos dentes na camada cementada da engrenagem);

$\mathrm{R}$ é a razão de carga (R), a dureza (HV) da superfície da camada cementada.

RETI [25] propôs que na cementação de um aço similar a um SAE 5115, tenha na superfície SAE 51100, SAE 5150 a 0,5 mm, e SAE 5115 a 1,6 mm. Na Figura 10(A) pode ser visto a distribuição esperada de carbono ao longo da camada cementada, e na 10(B) a sobreposição dos diagramas de transformação sob resfriamento contínuo desses três aços. Esse estudo de RETI [25] mostra a complexidade das interações presentes numa camada cementada, inclusive comportamento das tensões residuais, e que não ocorrem na análise de corpos-de-prova discretos como proposto no presente artigo.
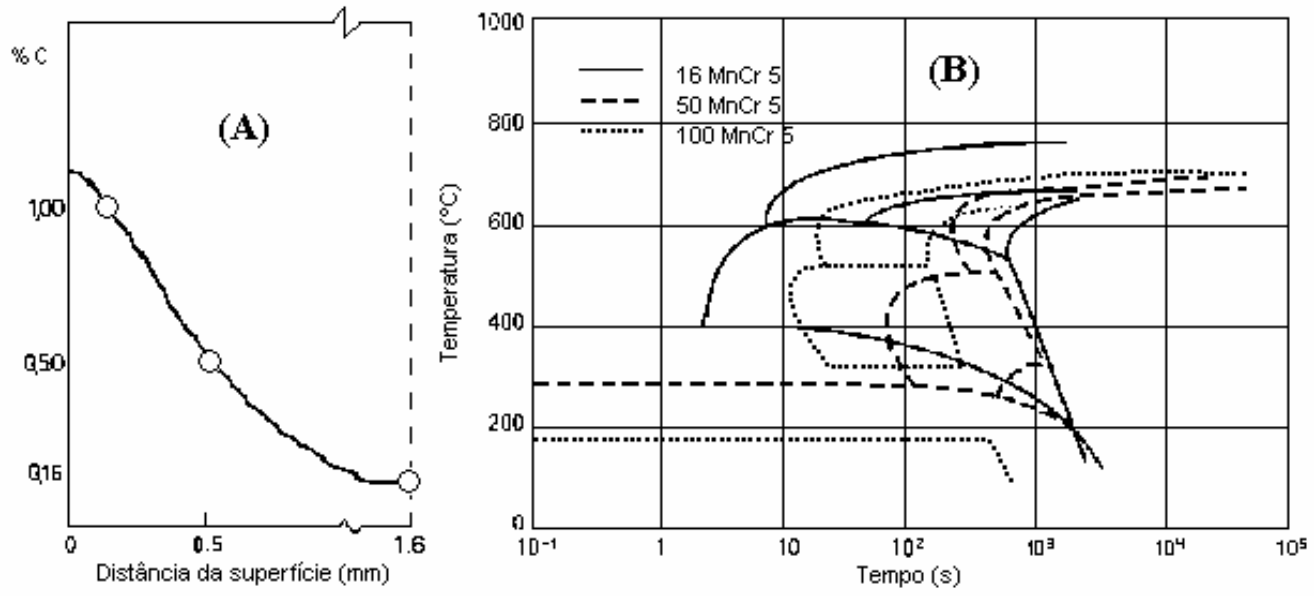

Figura 10: (A) Teor de carbono próximo à superfície e (B) sobreposição dos diagramas de transformação sob resfriamento contínuo dos aços SAE 5115, 5150 e 51100, segundo RETI [25].

\subsection{Fractografia}

As fractografias feitas com MEV das superfícies de fratura são mostradas nas Figuras 11 a 14 . Revelam aspecto dúctil no aço SAE 5115, e alternância de aspecto dúctil para frágil à medida que se vai do SAE 5140 para 5160 e 52100. Devido à grande fragilidade dos aços SAE 5140,5160 e 52100, que estão com dureza acima de $550 \mathrm{HV}$, torna-se difícil perceber as diferenças fractográficas na região de propagação de trinca por fadiga e na região de fratura final. Levando-se estas características para uma camada cementada, poderia ser interpretado como sendo o mecanismo de fratura intergranular ocorrendo na parte mais externa. A existência de aspecto alveolar mais pronunciado no aço SAE 52100 do que no aço SAE 5160, talvez seja devido ao fato do aço SAE 52100 ter carbono mais elevado, portanto, mais carbonetos pró-eutetóides que após a fratura revelariam um aspecto alveolar, mas não devido à ductilidade, mas talvez ao arrancamento dos carbonetos.

SHIN et al [26] fizeram estudo de similar a SAE 5115, mas com 0,30\% de Mo, comparando amostras cementadas em forno de atmosfera controlada, com amostras cementadas por plasma. Verificou que o modo de fratura do aço cementado por plasma foi predominantemente, enquanto que para o aço cementado em atmosfera controlada foi predominantemente intergranular com limite de fadiga mais baixo que no processo por plasma. 
Este estudo, por utilizar corpos-de-prova de vários aços diferentes, não leva em consideração as tensões residuais que variam ao longo da camada cementada, devido ao gradiente do teor de carbono na mesma. A transformação de austenita retida e a alteração resultante no volume relativo da camada e do núcleo são os principais responsáveis pelas alterações nos níveis de tensões residuais como uma função da temperatura. Como resultado da difusão do tratamento termo-químico, no caso da cementação, obtém-se uma camada superficial onde a distribuição do carbono é heterogênea, fazendo com que se tenha também uma distribuição heterogênea nas tensões residuais. Essas tensões não são consideradas nas equações (2) e (3) obtidas neste trabalho.

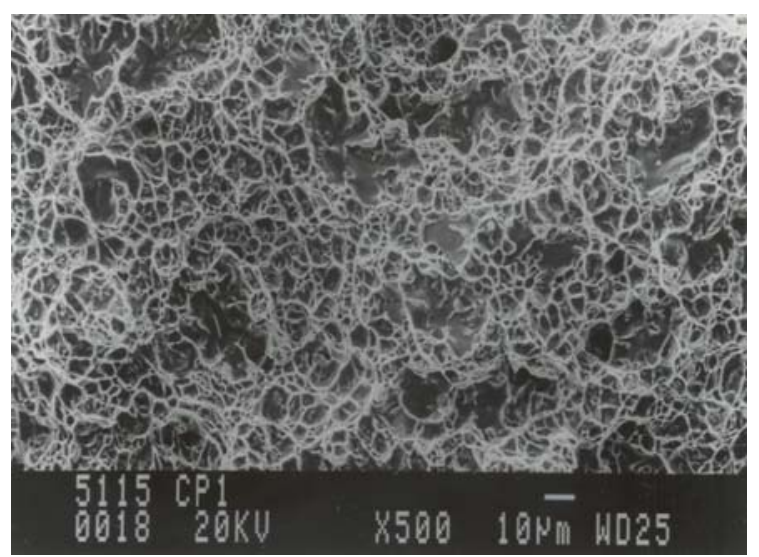

Figura 11: Fractografia dos corpos de prova de tenacidade à fratura para o aço SAE 5115. Região da fratura final, onde também predominam os alvéolos de fratura dúctil, sem mecanismo intergranular.



Figura 12: Fractografia dos corpos de prova de tenacidade à fratura para o aço SAE 5140. Região de fratura final, também com predominância de fratura alveolar, e poucas regiões com intergranular.

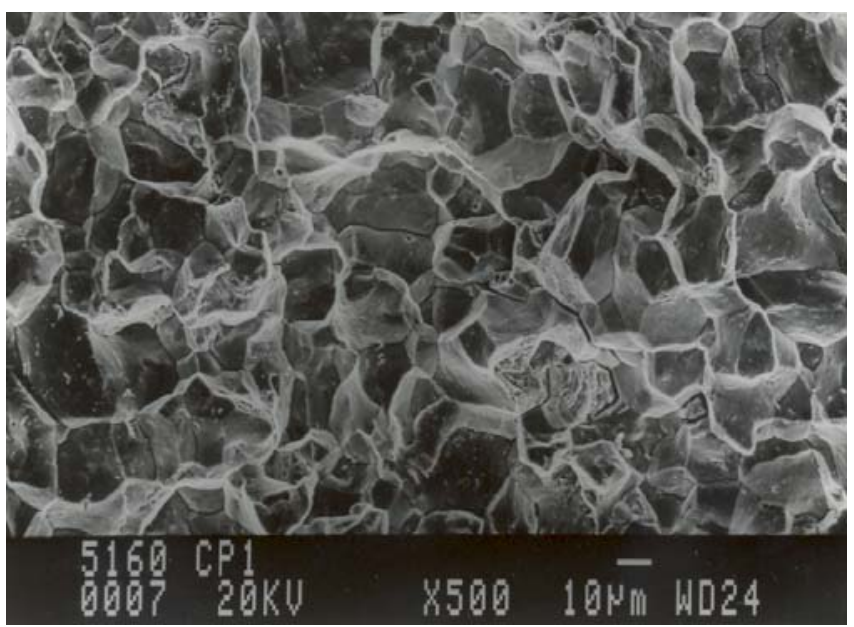


Figura 13: Fractografia dos corpos de prova de tenacidade à fratura para o aço SAE 5160. Região de fratura final, com predominância quase que total de fratura intergranular.

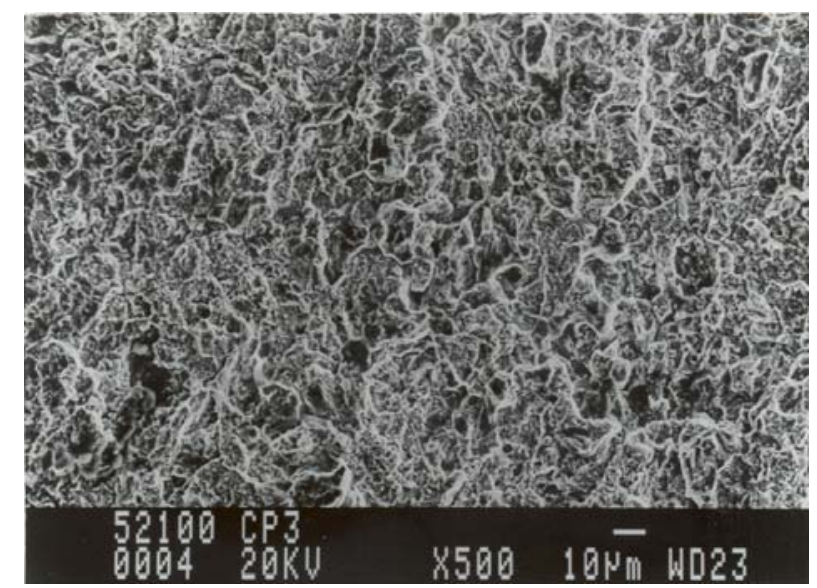

Figura 14: Fractografia dos corpos de prova de tenacidade à fratura para o aço SAE $52100 \mathrm{com} \mathrm{W}=10 \mathrm{~mm}$. Região de fratura final com fratura intergranular, intercalada com regiões de aspecto alveolar.

\section{CONCLUSÃO}

A revisão bibliográfica confirma que a camada cementada é uma região de grande complexidade para ser estudada, devido aos gradientes de carbono, propriedades mecânicas, microestrutura e tensões residuais que quando tem seus efeitos somados, dificultam a análise. Isto talvez justifique a maioria das referências sendo sobre fadiga e apenas 3 delas, LARSON [11], OBATA [19]] e PRESTON [22] serem relacionadas à mecânica da fratura.

Com o uso de amostras retiradas numa mesma família de aço com variação apenas do teor de carbono, é possível simular o comportamento da tenacidade à fratura ao longo de uma camada cementada, no caso em questão SAE 5115, 5140, 5160 e 52100 devido a falta do SAE 51100, Assume-se que apenas o teor de carbono varia de modo significativo. As durezas individuais dos corpos-de-prova coincidindo com a curva de dureza obtida após a cementação, têmpera e revenimento de engrenagens do mesmo aço SAE 5115, mostram que a metodologia proposta é consistente para analisar a tenacidade à fratura ao longo da camada cementada, mas sem considerar as tensões residuais de compressão.

Confirmou-se que a tenacidade à fratura varia de modo inversamente proporcional ao teor de carbono e microdureza, indicando que as equações propostas (2) e (3) podem ser utilizadas para previsão do comportamento da tenacidade à fratura de camada cementada em aços SAE 5115 ou similares. A facilidade de propagação de trinca diminui com o teor de carbono, indicando que uma trinca superficial pode ser paralisada ou retardada, quando durante sua propagação atingir um ponto que tenha um valor de $\mathrm{K}_{\mathrm{IC}}$ mais elevado. Esta observação vem também justificar a dificuldade de se conduzir teste de fadiga de modo estável em aços cementados, temperados e revenidos. . Estas equações propostas também podem ser utilizadas em conjunto com as equações dos demais autores comentados, permitindo maior previsão no comportamento de trincas.

As fractografias confirmaram a predominância do mecanismo de fratura intergranular para teores de carbono acima de 0,40 \%, como é esperado para aços de baixa liga cementados e temperados. Para o aço SAE 5115 predominou o micromecanismo de fratura alveolar. Para os aços SAE 5140, 5160 e 52100, verifica-se o micromecanismo predominantemente intergranular com uma certa quantidade de alvéolos sendo que o percentual de cada micromecanismo observado (intergranular ou alveolar) está associado à austenita retida transformada em fase mais dúctil durante o revenimento, ou carbonetos esféricos arrancados durante à propagação da trinca no corpo de prova de tenacidade à fratura.

\section{BIBLIOGRAFIA}

[1] AMERICAN SOCIETY FOR TESTING AND MATERIALS, ASTM E399-91, Standard Test Method for Plane-Strain Fracture Toughness of Metallic Materials, West Conshohocken, 1997. 
[2] AMERICAN SOCIETY FOR TESTING AND MATERIALS, ASTM E1290-02, Standard Test Method for Crack-Tip Opening Displacement (CTOD) Fracture Toughness Measurement. West Conshohocken, 2002.

[3] AMERICAN SOCIETY FOR TESTING AND MATERIALS, ASTM E1820-01, Standard Test Method for Measurement of Fracture Toughness, West Conshohocken, 2001.

[4] AMERICAN SOCIETY FOR TESTING AND MATERIALS, ASTM E8-01, Standard Test Methods for Tension Testing of Metallic Materials, West Conshohocken, 2001.

[5] UNTERWEISER, P.M., BOYER, H.E., KUBBS, J.J., "Heat Treater’s Guide”, American Society for Metals, ed. 4, Ohio, pp. 135, 193, 188, 195, 198, 201, 204, 206, 1987.

[6] AMERICAN SOCIETY FOR TESTING AND MATERIALS, ASTM E92-82, "Standard test Method of Vickers”, Hardness of Metallic Materials, West Conshohocken, pp. 31, 1982.

[7] AMERICAN SOCIETY FOR TESTING AND MATERIALS, ASTM E3-01, "Standard Practice of Preparation of Metallographic Specimens”, West Conshohocken, pp. 26, 2001.

[8] SAE - SOCIETY OF AUTOMOTIVE ENGINEERS - HANDBOOK V.1., Materials, Fuels, Emissions and Noise. SAE J406 (Mai98) - "Methods of Determining Hadernability of Steels", Table A15 Hadernability multiplyng factors vs \% element. 1.43, Warrendale, pp.140, 1998.

[9] HUME-ROTHERY, W., “Estrutura das Ligas de Ferro”, São Paulo, editora Edgar Blücher Ltda, pp 168169, 1968.

[10] REED-HILL, R.E., “Princípios de Metalurgia Física”, Rio de Janeiro, editora Guanabara Dois S. A., pp. 610-618, 1982.

[11] LARSSON, M., ÖULUND, P., BLOM, R., WALBURGUER, H., MELANDER, A., PRESTON, S., "Fatigue Properties After Carburizing Influence of Core Hardness and Notch Geometry on Fatigue Properties of Case Hardened Steels”, Scandinavian Journal of Metallurgy, v. 23, n. 2, pp.62-73, 1994.

[12] SAE - SOCIETY OF AUTOMOTIVE ENGINEERS - HANDBOOK V.1., Materials, Fuels, Emissions and Noise SAE J404, “Chemical compositions of SAE alloy steels”, Warrendale, pp. 3, 2000.

[13] AMERICAN SOCIETY FOR METALS INTERNATIONAL. ASM Handbook, Volume 1, "Properties and Selection: Irons, Steels, and High-Performance Alloys”, v. 11, ASM International, 2000.

[14] ATKINS, M., “Atlas of Continous Cooling Transformation Diagrams for Engineering Steels”, ASM, Ohio, 1980.

[15] NAGAMURA, K., TERAUCHI, Y., MATOWIBOWO, S.Y., "Study on Gear Bending Fatigue Strength Design Based on Reliability Engineering (Prediction of Crack Propagation and Fatigue Life of MAC14 Supercarburized Steel Gear)”, JSME International Journal, Series C, v. 37, n. 4, pp. 795803, 1994.

[16] SHIN, J.C., LEE, S., RYU, J. H., “Correlation of Microstructure and Fatigue Properties of Two HighStrength Spring Steels”, International Journal of Fatigue, v. 21, pp. 571-579, 1999.

[17] ERDOGAN, M., TEKELI, S., "The Effect of Martensite Particle Size on Tensile Fracture of SurfaceCarburized AISI 8620 Steel with Dual Phase Core Microstructure”, Materials and Design, v. 23, n. 7, pp. 597-604, 2002.

[18] DIN DEUTSCHES INSTITUT FÜR NORMUNG. DIN EN ISO 2639 "Determination and Verification of the Depth of Carburized and Hardened Case”, Berlim, pp. 11, 2002. 
[19] OBATA, Y., KOBAYASHI, H., AOKI, K.I., YAMAGUCHI, T., SHIBATA, K., “Evaluation of Fatigue Crack Growth Rate of Carburized Gear by Acoustic Emission Technique”, ASTM Special Technical Publication, n. 1077, pp. 261-270, 1991.

[20] COHEN, R.E., MATLOCK, D.K., KRAUSS, G., "Specimen Edge Effects on Bending Fatigue of Carburized Steel”, Journal of Materials Engineering and Performance, v. 1, n. 5, pp. 695-704, 1992.

[21] KATO, M., INOUE, K., DENG, G, JEONG, B.S., "Size Effect in Bending Strength of Carburized Gear Teeth”, American Society of Mechanical Engineers, Design Engineering Division (Publication) DE, v. 43, Advancing Power Transmission Into the 21st Century, pp 661-668, 1992.

[22] PRESTON, S., "Fatigue Crack Initiation and Growth from a Gas Carburized Surface”, Materials Transactions, JIM, v. 34, n. 1, pp. 27-32, 1993.

[23] GENEL, K., DEMIRKOL, M., "Effect of Depth on Fatigue Performance of AISI 8620 Carburized Steel”, International Journal of Fatigue, v. 21, pp. 207-212, 1999.

[24] MASUYAMA, T., KATO, M., INOUE, K., YAMASHITA, T., "Evaluation of Bending Strength of Carburized Gears Based on a Quantification of Defect Size in The Surface Layer”, Journal of Mechanical Design, Transactions of the ASME, v. 124, n. 3, pp. 533-538, 2002.

[25] RETI, T., "Residual Stresses in Carburised, Carbonitrided and Case-hardened Components (Part 1)”, Heat Treatment of Metals, v. 30, n. 4, pp. 83-96, 2003.

[26] SHIN, D.M., LEE, C.Y., LEE, K.S., "Fatigue Properties of Plasma Carburized Low Carbon Cr-Mo Steel”, ISIJ International, v. 42, n. 9, pp. 1042-1048, 2002. 